\title{
ENSEÑANZA - APRENDIZAJE DEL INGLÉS BAJO LAS POLÍTICAS DEL MINISTERIO DE EDUCACIÓN EN COLOMBIA:
}

\section{VOCES DESDE EL AULA DE CLASEEFL TEACHING AND LEARNING UNDER THE MINISTRY OF EDUCATION POLICIES IN COLOMBIA: VOICES FROM THE CLASSROOM}

\author{
Margarita M. López Pinzón1 \\ Odilia Ramírez Contreras ${ }^{2}$ \\ Mauricio Arango Vélez ${ }^{3}$ \\ Universidad de Caldas, Manizales, Colombia
}

\section{RESUMEN}

Las políticas relacionadas con la enseñanzaaprendizaje del inglés como lengua extranjera

$1 \quad$ Margarita López holds an MA in English Didactics from Universidad de Caldas (Colombia) and is currently the Director of this graduate program. She has extensive experience in teaching graduate and undergraduate courses and has been conducting educational research for several years. Her areas of interest include EFL methodogy, research and bilingualism. margarita.lopez@ucaldas.edu.co

3203474426 Universidad de Caldas Manizales MARGARITA LOPEZ: 0000-0002-6755-3764

2 Odilia Ramírez has been a research professor and adviser at Universidad de Caldas (Colombia) for 10 years. She is also a public school principal in Manizales with long experience in EFL teaching and research. She holds a $B A$ in modern languages and MA in English Didactics from the same university. odiliaramirez@hotmail.com 311748 9757 ODILIA RAMIREZ: 0000-0002-8075-3381

3 Mauricio Arango holds an MA in English Didactics from Universidad de Caldas in Manizales (Colombia). His teaching experience includes language centers, social-academic projects, colleges and teacher training for national and local bilingualism projects. His main interest is the design and implementation of ELT material for both in-person and online classes aimed to enhance students' engagement and foster intercultural competence at the same time. arangomauenr@gmail.com 3113942820 Universidad de Caldas Manizales, Colombia MAURICIO ARANGO: 0000-0001-83755078 en Colombia han sido un área de mucho interés en el campo educativo en los últimos años. Este artículo presenta los resultados de un estudio de tipo mixto realizado en cinco regiones de Colombia para describir las percepciones de profesores, estudiantes y directivos sobre la enseñanza y aprendizaje del inglés en colegios públicos bajo los lineamientos del Ministerio de Educación, específicamente el Currículo Sugerido (CSI) y los Derechos Básicos de Aprendizaje (DBA). Los participantes fueron 1526 estudiantes de grados $10^{\circ}$ y $11^{\circ}$, además de 61 profesores de inglés y 22 coordinadores de 26 instituciones educativas públicas. A través de encuestas y entrevistas semiestructuradas se recopiló información cualitativa y cuantitativa. Los resultados sugieren que las Instituciones Educativas (IE) focalizadas por el Programa Colombia Bilingüe han hecho esfuerzos considerables para adaptar sus currículos incorporando en ellos el CSI y los DBA; los participantes reportaron percepciones 
positivas acerca de la implementación de estas políticas en lo relacionado con el uso apropiado de los enfoques metodológicos, los materiales y recursos, los contenidos temáticos y las estrategias de evaluación en sus aulas de clase; sin embargo, también sostuvieron que se requiere darle continuidad a los programas de bilingüismo para alcanzar los objetivos de nación en esta asignatura y recibir apoyo permanente de las autoridades locales y nacionales tanto en inversión como en acompañamiento pedagógico pues estas políticas se consideran una forma de brindar a los estudiantes la oportunidad mejorar los niveles de proficiencia en inglés en el nivel de educación básica secundaria y media.

\section{PALABRAS CLAVES:}

Derechos Básicos de Aprendizaje (DBA), enseñanza del inglés, aprendizaje del inglés, Currículo sugerido de inglés (CSI), políticas educativas.

ABSTRACT

Policies concerning the teaching and learning of EFL in Colombia are an area of interest in the field of education in recent years. This article presents the results of a mixed methods study conducted in five Colombian regions to describe the views of teachers, students, and administrators about EFL teaching and learning in public schools under the Suggested English Curriculum (SEC) and Basic Learning Rights (BLR). Participants included 1526 students from $10^{\text {th }}$ and $11^{\text {th }}$ grades, 61 English language teachers, and 22 coordinators from 26 schools. This was a mixed methods study, in which both quantitative and qualitative data was collected and analyzed from questionnaires and semistructured interviews. The results suggest that focalized schools made a considerable effort to adapt their curricular designs to the SEC and BLR guidelines. Participants' perceptions about the SEC and BLR implementation were positive and focused on the proper use of the methodological approaches, materials and resources, curricular themes, and assessment procedures in their EFL classes. However, they claimed that continuity of bilingualism programs is required to achieve the proposed goals. We conclude that to keep strengthening the ELF teaching-learning process in the country, schools need constant support from educational authorities at all levels. Teachers are encouraged to provide students with lessons that follow the SEC and BLR guidelines and consider their linguistic level, needs, interests and contexts at the same time.

\section{KEYWORDS:}

Basic Learning Rights (BLR), EFL teaching, EFL learning, mixed methods, Suggested English Curriculum (SEC)

\section{INTRODUCCIÓN}

En el contexto colombiano el Ministerio de Educación (MEN) le ha dado prioridad a la enseñanza del inglés como lengua extranjera (EFL) y ha dirigido sus esfuerzos al desarrollo de la competencia comunicativa a través del Programa Nacional de Bilingüismo (PNB) (MEN, 2014a). A pesar de las críticas a la dicotomía español-inglés seleccionada por el gobierno nacional para referirse al bilingüismo en el Sistema educativo (Truscott, 2006, 2012; Bonilla \& Tejada, 2019). Las razones para determinar tal prioridad al aprendizaje del inglés incluye (1) el papel de la globalización en la discusión y análisis de los temas relacionados con derechos humanos, protección ambiental, equidad social, cultura y tecnologías de la información y comunicaciones (TIC), las cuales, a su vez, conllevan a promover una interacción constante entre los ámbitos locales y globales y (2) la prevalencia del inglés con respecto al acceso del conocimiento, ya sea textos académicos o científicos o la alta cantidad de información disponible en línea (MEN, 2014a). 
De igual manera, el MEN ha considerado la relación entre la competencia en inglés y el desarrollo propuesto por Coleman (como se cita en MEN, 2014a), ya que la competencia en inglés tiene un impacto positivo en el acceso y mejoramiento de las oportunidades laborales, cooperación internacional, información y estudios de investigación y movilidad estudiantil (MEN, 2014a). Dos de las iniciativas más promovidas dentro del PNB son el Currículo Sugerido de inglés (CSI) y los Derechos Básicos de Aprendizaje (DBA) emitidos en el 2016. Estos documentos se presentaron para guiar a los docentes en la construcción de un currículo local contextualizado. La estructura del CSI incluye cuatro componentes principales: alcance y secuencia que establece las metas, funciones del idioma y los objetivos para ser alcanzados en cada grado escolar; los componentes del currículo que articula los contenidos con funciones dadas y los objetivos que proponen los indicadores de desempeño sugeridos; la estructura metodológica sugerida que propone el Aprendizaje Basado en Tareas, Aprendizaje Basado en Proyectos y Aprendizaje Basado en Problemas como los enfoques principales para ser implementados gradualmente desde el grado sexto hasta el grado once. Finalmente, el enfoque sugerido de evaluación que sugiere los procedimientos para monitorear los avance en el aprendizaje (MEN, 2016). Por otro lado, los DBA enumeran las habilidades a ser desarrolladas y adquiridas en cada grado escolar. Los DBA también incluyen estrategias pedagógicas para contextualizar el aprendizaje con ejemplos que ilustran la habilidad dada (MEN, 2016).

En contraste, otras opiniones conciben las iniciativas gubernamentales como poco realistas y ambiciosas. González (2010), por ejemplo, menciona las críticas a la adopción del Marco Común Europeo de Referencia (MCER) como un parámetro del idioma ya que este fue diseñado para un contexto social, económico y cultural diferente. Además, Cárdenas (como se cita en González, 2010) argumenta que el MCER "debió haber sido contrastado con las condiciones de las Instituciones Educativas (IE) colombianas tales como la infraestructura, organización del currículo, el uso de los idiomas extranjeros en los ámbitos académicos y culturales del país (p. 342). González (2010) continúa preguntándose la manera cómo los docentes colombianos son responsables del éxito del programa nacional de bilingüismo y cómo el desarrollo profesional y la certificación docente pueden "convertirse en instrumentos de exclusión, inequidad, estandarización y comercialización en la formación docente" ( $p$. 342). Fandiño, Bermúdez and Lugo (2012) ratificaron esas opiniones enfatizando el hecho de que, por ejemplo, los docentes con alto nivel de competencia tal como C1 o B2 de acuerdo al MCER no deberían ser culpados por obtener resultados bajos de competencia ya que ellos se enfrentan a clases con un alto número de estudiantes, recursos limitados, restricciones de tiempo o curriculares, entre otros aspectos. Usma (2009) también advirtió que aunque el PNB y sus políticas de idiomas asociadas conllevan a oportunidades para que el país participe en la escena internacional éstas "generan principalmente inequidad, exclusión y estratificación con los nuevos discursos y prácticas que se adoptan" (p. 134), lo cual, a su vez, se traduce en el fortalecimiento de las diferencias sociales que también fueron discutidas por Mackenzie (2019) y Bonilla and Tejada (2016) quien resaltó el hecho de que la mayoría de las veces los beneficios del PNB están relacionados con aspectos socioeconómicos tales como la competitividad y acceso a las oportunidades laborales.

Con el fin de determinar cómo estas dos visiones contrastan, este artículo presenta el estado del arte, desde la perspectiva de los estudiantes, docentes y directivos de la enseñanza y aprendizaje del inglés en IE públicas bajo el Currículo Sugerido (CS) y los Derechos Básicos 
de Aprendizaje (DBA) en cinco regiones de Colombia: Cafetera, Norte, Centro, Pacifica y Noroeste. Específicamente, el estudio se enfocó en cómo los participantes perciben el CS y los DBA como parte del actual PNB cuyo propósito, de acuerdo al MEN, es acortar la distancia del acceso a las oportunidades de aprendizaje del idioma inglés entre los estudiantes de IE públicas y privadas (MEN, 2016).

Aunque en países tales como Australia, Canadá y los Estados Unidos los programas de bilingüismo se han implementado desde la década de los 70 (Sánchez \& Obando, 2008), en Colombia esta clase de programa se pueden considerar reciente. Esta es la razón por la cual para determinar científica y sistemáticamente cómo esas políticas de educación se perciben por las partes involucradas en el proceso en las aulas de clase reales, se desarrolló un método mixto (Creswell, 2018) para explorar las opiniones de los estudiantes, docentes y coordinadores. Las preguntas de investigación que guiaron el estudio fueron: (1) ¿Cómo perciben los miembros de la comunidad educativa de cinco regiones colombianas el CSI y los DBA como parte del programa de bilingüismo y (2) ¿Cómo se han incorporado el CSI y los DBA en la practicas pedagógicas en las aulas de clase de inglés en cinco regiones colombianas?

\section{BILINGÜISMO EN COLOMBIA}

El concepto de bilingüismo y la forma como éste se ha incorporado en el contexto de la enseñanza del inglés como lengua extranjera (EFL) en Colombia, ha sido materia de discusión; Fandiño and Jiménez (2012) han señalado que definir el termino bilingüismo no ha sido tarea fácil y que autores de diversas disciplinas han tratado de clarificar este concepto. Algunos de los factores considerados para definir bilingüismo han sido los lingüísticos, sociológicos, políticos, psicológicos y pedagógicos. Con el fin de presentar y desarrollar un programa de bilingüismo, se han considerado varias definiciones que están alineadas con la competencia lingüística, los objetivos del programa así como los contextos, pero en el caso específico del MEN en Colombia y dentro del contexto de este estudio, el término se define como "los diferentes grados de fluidez con los cuales un individuo es capaz de comunicarse en más de un idioma y cultura y esos grados dependen del contexto en el cual cada persona se desenvuelve" (MEN, 2006, $p$ 5 , traducción de los autores); esto significa que los niveles de competencia de los estudiantes de EFL pueden variar de acuerdo a sus propios factores de contexto. Por el contrario, Grosjean (como se cita en Bakić \& Škifić, 2017) define el bilingüismo como "el uso regular de dos o más idiomas (o dialectos) en su vida diaria" (p.35) lo cual también implica la Importancia del contexto en el desempeño del hablante. Liebkind (como se cita en Bakić \& Škifić, 2017) propone varias condiciones para considerar a una persona bilingüe, además de que "los criterios de origen, competencia en el idioma y la función del idioma son condiciones cruciales al igual que las actitudes" (p. 35), es decir que el dominio emocional (sentimientos, actitudes, emociones) del estudiante de idiomas determina de alguna manera sus características bilingües.

Con respecto al contexto colombiano, Sánchez and Obando (2008) reportaron su análisis de la implementación del CBP con un examen de los principales requisitos académicos necesarios para alcanzar los objetivos de dicho programa. Los autores resumieron una explicación cronológica de las acciones tomadas por el MEN desde la Ley General de Educación (MEN, 1994), la Resolución 2343 (MEN, 1996), y la Ley 715 (MEN, 2001) hasta los Estándares Básicos de Competencia para el Idioma Extranjero: Inglés (MEN, 2006) advirtiendo que los docentes y los estudiantes no podrían estar totalmente consientes de las metas que busca MEN; lo cual hace más difícil cumplir con los políticas establecidas y los lineamientos curriculares 
como resultado de la falta de información ya que en Colombia "las normas son creadas en un enfoque de 'arriba hacia abajo' en el cual pocas personas (no necesariamente docentes) saben que 'deben hacer y por qué' y además la participación de los docentes en la creación de estándares es prácticamente invisible" (Sánchez \& Obando, 2008, p. 184). De igual manera, Gómez (2017) revisó los antecedentes del bilingüismo en Colombia, los planes y las políticas lanzadas entre 2004 y 2016 y afirma que debido a que los docentes son los que implementan las metodologías y estrategias, ellos son los que necesitan estar más involucrados en el proceso de toma de decisiones y evaluación de los planes de bilingüismo, además de que se deben considerar condiciones locales tales con el número de estudiantes por clase, los materiales disponibles, el uso de la lengua extranjera en clase, entre otras.

\section{PROGRAMA COLOMBIA BILINGÜE}

204 EI PCB surgió basado en dos propósitos: (1) darle prioridad al fortalecimiento de la enseñanza del inglés como una asignatura escolar durante diez años y (2) consolidar el proceso enseñanzaaprendizaje haciendo uso efectivo de las horas dictadas en las IE (MEN, 2016b). Este programa estableció como meta principal que el $50 \%$ de los estudiantes de grado 11 alcanzaran el nivel B1 del MCER al 2025. PCB (2014-2018) se enmarcó inicialmente con el propósito de mejorar el aprendizaje del idioma inglés en Colombia y sus estrategias iniciales se centraron en 41 Secretarias de Educación, 1.400 docentes y 128.000 estudiantes (MEN, 2015). En el año 2016, MEN afirmó que Colombia había tenido un avance significativo en el fortalecimiento de los procesos de enseñanza-aprendizaje del inglés, aunque los resultados en los exámenes nacionales estandarizados muestran que la mayoría de la población no se puede comunicar en este idioma. La razón puede ser que el inglés no era una prioridad en el sistema educativo nacional y por lo tanto el MEN intentó brindarle a todos los niños, niñas y adolescentes matriculados en planteles educativos públicos la oportunidad de aprender inglés, sin embargo, esto ha generado un debate acerca de la falta de una base social en el PCB ya que un amplio sector de la comunidad académica apoya la idea de que el inglés fue impuesto y los docentes y estudiantes han sido forzados a ensenar y aprender este idioma extranjero sin una necesidad razonable. Por ejemplo, Herazo at al. (2012) proporciona evidencia de que "el mercado laboral colombiano rara vez requiere de competencia en inglés para conceder un puesto laboral." (p. 206).

Desde el año 2015, se han promovido varias estrategias centradas en los estudiantes, docentes, IE públicas y secretarías de educación (MEN, 2018). De hecho, el PCB ha actuado en diferentes aspectos tales como capacitación docente, planes de incentivo docente, diagnóstico del nivel de inglés de los docentes, distribución de materiales didácticos y textos escolares y la creación de modelos o enfoques pedagógicos curriculares. En este sentido, los objetivos del PCB se resumen en tres líneas estratégicas: Capacitación, Materiales y Monitores (MEN, 2016b).

Para la implementacion del programa se promovieron tres iniciativas: Pimero, el Programa de Formadores Nativos Extranjeros (FNE) en el cual hablantes nativos de inglés fueron invitados a las aulas de clase de las IE públicas con el fin de interactuar con los estudiantes y coenseñar con los docentes colombianos. Es importante resaltar que los FNE llegaron desde una gran variedad de paises de habla inglesa y su condición de nativos extranjeros estaba conectada al país y cultura que representaban, no necesariamente a la cultura tradicional occidental que Holiday (2009) ha criticado como una fuerza divisoria que sugiere "una cultura superior de ensenanza y aprendizaje" 
(386); segundo, programas de incentivos en inmersiones nacionales e internacionales en La Tebaida (Colombia), los Estados Unidos, India, y el Reino Unido (MEN, 2018); tercero, el desarrollo profesional basicamente para proporcionarle a los docentes las habilidades necesarias para incorporar el CSI y los BLR en su planeación al igual que el uso de materiales variados brindados por el MEN.

Con respecto a los materiales, los objetivos del programa se establecieron para desarrollar un curriculo general, proporcionar lineamientos para el diseño del curriculo EFL y el desarrollo y publicacion de materiales para apoyar el aprendizaje del inglés desde el grado $6^{\circ}$ hasta el grado $11^{\circ}$. En enero del 2016, las 11.500 IE públicas de Colombia recibieron 22.000 Colombia Bilingüe English Kits (CBEK) los cuales incluían los siguientes documentos: el Curricular Sugerido de inglés, Los Derechos Básicos de Aprendizaje, Los Lineamientos y Principios Pedagógicos, y La Guía No 22: Estándares Básicos de Competencia en un Idioma Extranjero. De esta manera, los docentes recibieron herramientas para definir las metas y los objetivos de aprendizaje teniendo en cuenta sus contextos locales y regionales, así como para el Desarrollo de proyectos, tareas, exámenes y materiales que promuevan el proceso de aprendizaje EFL (MEN, 2018). Así mismo, se brindaron materiales de texto para usar en el aula de clase tales como Way to go! e English, please! que fueron dos series de textos escolares y libros de talleres que recibieron las IE para el trabajo individual de los estudiantes (MEN, 2016). Finalmente, el PCB diseño un programa de seguimiento para evaluar las acciones implementadas en las IE focalizadas desde el 2016 hasta el 2018 que consistió en un apoyo al proceso in situ y que contó con la participación de los estudiantes, docentes, personal directivo, padres de familia y autoridades locales de educación (MEN, 2016a) al igual que con un grupo de 15 profesionales conocidos como Gestores de Bilingüismo.

\section{METODOLOGÍA}

Por su naturaleza descriptiva, el presente estudio se enmarcó dentro del enfoque de Métodos Mixtos propuesto por Creswell (2018). Se seleccionó el diseño convergente o concurrente que le permitió a los investigadores reunir los resultados del análisis de datos cuantitativos y cualitativos y compararlos o combinarlos (Creswell 2009). Para la recolección de datos de este estudio los investigadores utilizaron una encuesta para estudiantes de grado $10^{\circ}$ y $11^{\circ}$ (Anexo 1), una encuesta para docentes de inglés (Anexo 2) y un protocolo de entrevista para directivos (rectores o coordinadores) (Anexo 3).

El proceso de recolección de datos incluyó visitas presenciales a 26 IE cuyos rectores se contactaron a través de correo convencional y electrónico para obtener su consentimiento informado de participación en la investigación (Burns, 2001). Los datos fueron recolectados por medio de cuestionarios impresos para los estudiantes y la mayoría de los docentes cuyas aulas se visitaron para la administración de los mismos, se complementó la muestra por medio de cuestionarios digitales para algunos docentes y se utilizó un protocolo de entrevista semiestructuradas las cuales fueron llevadas a cabo con rectores, coordinadores y jefes del área de inglés. Los instrumentos fueron diseñados por los investigadores con el propósito de obtener información acerca de las percepciones de los estudiantes, docentes y directivos en cuanto a la implementación de CSI y los DBA. Las preguntas de la encuesta y la entrevista se dividieron en cinco secciones principales que permitieron explorar los siguientes aspectos del proceso de enseñanza y aprendizaje en el aula de clase EFL junto con información demográfica: motivación, metodología, materiales, contenido o temas y tipos de evaluación; una última sección incluyó dos preguntas abiertas para obtener comentarios adicionales y sugerencias acerca 
del CSI y los DBA por parte de los participantes Los datos fueron procesados usando la herramienta tecnológica Quia y las entrevistas de los coordinadores fueron grabadas, transcritas y categorizadas; coherentes con el enfoque de método mixto y sus principios de validez y confiabilidad, se realizó el análisis de datos por medio de la triangulación (Freeman, 1998; Burns, 2001; Creswell, 2009). La interpretación incluyo la búsqueda de resultados cuantitativos y cualitativos y la evaluación de cómo la información abordo las preguntas de investigación,

\section{PARTICIPANTES}

Este estudio incluyó estudiantes, docentes y rectores/coordinadores de $26 \mathrm{IE}$ localizadas en nueve ciudades colombianas en cinco regiones diferentes: Manizales y Medellín (Región Cafetera), Montería y Cartagena (Región Norte), Neiva (Región Centro), Cali y Palmira (Región Pacifico), and Bucaramanga

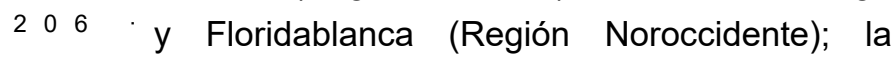

selección de los participantes se llevó a cabo por medio del muestreo intencional (Trochim \& Donnelly, 2008) ya que todos ellos fueron parte del sistema educativo público con los roles de estudiantes, docentes y directivos (rectores/ coordinadores) directamente relacionados con la implementación del CSI y DBA. El criterio de selección incluyo los siguientes aspectos: IE pertenecientes al PCB como IE focalizadas en todo el país; en cada ciudad se visitaron un promedio de tres instituciones localizadas estratégicamente en vecindarios de clase baja y media. Los docentes y estudiantes pertenecieron a los grados $10^{\circ}$ y $11^{\circ}$ grado teniendo en cuenta su exposición al CSI y DBA durante los últimos dos años. Además, los coordinadores fueron seleccionados como parte de la administración del currículo escolar que estuvieron a cargo del seguimiento del proceso de enseñanza EFL en las IE mencionadas. El total de participantes en el estudio fue de 1526 estudiantes de los grados 10 y 11,61 docentes de inglés y 22 coordinadores tal como se resume en la Tabla 1.

Tabla 1. Número de participantes en el estudio de acuerdo con la región

\begin{tabular}{|c|c|c|c|c|c|c|c|}
\hline \multicolumn{2}{|c|}{ Región } & Cafetera & Norte & Centro & Pacifico & Noreste & Total \\
\hline \multirow{2}{*}{ Estudiantes } & $10^{\circ}$ & 201 & 181 & 80 & 169 & 139 & $\mathbf{7 7 0}$ \\
\cline { 2 - 8 } & $11^{\circ}$ & 168 & 193 & 103 & 140 & 152 & $\mathbf{7 5 6}$ \\
\hline \multicolumn{2}{|c|}{ Docentes } & 21 & 15 & 7 & 8 & 10 & $\mathbf{6 1}$ \\
\hline \multicolumn{2}{|c|}{ Rectores / Coordinadores } & 5 & 7 & 2 & 6 & 2 & $\mathbf{2 2}$ \\
\hline
\end{tabular}

La encuesta de los estudiantes fue administrada a 1.526 estudiantes de $10^{\circ}(51 \%)$ y $11^{\circ}(49 \%)$ grados; $54 \%$ de ellos fueron mujeres y $46 \%$ hombres con edades entre los 14 y 19 años. Con respecto a los docentes, la información demográfica reveló que el $37 \%$ estaba entre los 30 y 39 años, $27 \%$ entre los 40 y 49 y $27 \%$ entre los 50 y 59 años, lo cual significa que solamente el $9 \%$ eran mayores de 60 o menores de 29 . Entre ellos, 11 contaban con títulos de pregrado, la mayoría en Lenguas Modernas, 47 contaban con un posgrado (especialización o maestría) y 3 de ellos con doctorado en educación.

\section{RESULTADOS Y DISCUSIÓN}

Después de analizar y de realizar una triangulación de todos los datos arrojados por los instrumentos, los siguientes resultados reportan las percepciones de los participantes en cuanto a y al Currículo Sugerido de inglés y a los Derechos Básicos de Aprendizaje en el 
proceso de enseñanza y aprendizaje del inglés como lengua extranjera en Colombia.

\section{ACEPTACIÓN DE LOS ENFOQUES METODOLÓGICOS PROPUESTOS POR EL SEC}

En cuanto a la metodología de la enseñanza del inglés, un alto número de estudiantes $(65 \%)$ indicó estar familiarizado con el enfoque por tareas (TBL) pues reportaron que éste se implementaba con bastante frecuencia en sus clases mientras que otros resaltaron el enfoque por proyectos (PBL) (21\%) y el enfoque por resolución de problemas (PSBL) (14\%). Los participantes mencionaron las bondades de los enfoques y describieron cómo se combinaban con estrategias propias de la didáctica de la enseñanza del inglés como juegos de rol, reportes orales y ejercicios para completar información. En general, la metodología fue descrita como muy interesante y particularmente dijeron que con la implementación del CSI sus clases se volvieron más dinámicas e interesantes pues en ellas se trataron temas relevantes a su contexto. Con respecto a los DBA, los estudiantes manifestaron que desde la implementación del $\mathrm{CSI}$, los docentes los presentaban antes de que las clases comenzaran lo que les ayudaba a focalizar su atención y a darle una orientación específica a cada lección, lo cual les ayudó a estar más conscientes de los objetivos comunicativos a ser alcanzados en su grado escolar y a las actividades a ser desarrolladas en un periodo académico especifico. Asimismo, los docentes y directivos afirmaron que efectivamente como resultado de las capacitaciones sobre el CSI se había convertido en una práctica permanente en los colegios presentar los DBA antes de que las clases comenzaran, con el fin de hacer a los estudiantes más conscientes de las habilidades a ser desarrolladas en sus clases tal como lo expresan los siguientes comentarios tomados de las encuestas y entrevistas:
Compartir los DBA, le permitió a los estudiantes ser conscientes de lo que ellos deben aprender en clase. (DI)

Los Derechos Básicos de Aprendizaje nos ayudan a alcanzar los objetivos dela clase. (E)

El Currículo Sugerido de Ingles ha contribuido a que las clases de inglés sean más organizadas y productivas. (C)

Los participantes insistieron en que gracias al CSI y los DBA la metodología se volvió más variadas pues se llevaron a la práctica algunos modelos de interacción de clase y prácticas metodológicas específicas sugeridas, principalmente por tareas y proyectos en las aulas de clase teniendo presente tanto la función comunicativa como los componentes estructurales del idioma extranjero, Estos resultados también muestran sus puntos de vista acerca de la importancia de los tres enfoques metodológicos que deben ser usados para implementar el CSI. La mayoría de las IE compartieron los mismos procedimientos para dar las clases: primero, seleccionan algunos temas conectados a las unidades de su texto guía; luego definen el vocabulario; las funciones del idioma y las estructuras gramaticales considerando el conocimiento previo de los estudiantes y finalmente, proponen tareas en los grados $6^{\circ}$ y $7^{\circ}$ y proyectos en los grados $8^{\circ}$ y $9^{\circ}$ y en menor medida, trabajos para la resolución de problemas en grados $10^{\circ}$ y $11^{\circ}$.

En relación con la práctica docente y el apoyo con el programa FNE, los estudiantes, docentes y directivos estuvieron de acuerdo en que éstos enriquecieron el proceso enseñanza-aprendizaje del inglés con una planeación de clases contextualizada; la secuencia más frecuente descrita por los participantes es la siguiente: se llevó a cabo una actividad de preparación, luego los FNE propusieron actividades de 
compresión auditiva y actividades de habla y los docentes llevaron a cabo ejercicios de escritura y lectura, para después intercambiar grupos. Un alto número participantes expresó que la metodología de los nativos fue buena y que ellos esperaban contar con mas en el futuro para que les continuaran fortaleciendo su habilidad de habla, tal como lo indica el siguiente testimonio:

El Ministerio de Educación debe invertir más en los docentes nativos de tal manera que ellos ayuden a mejorar aspectos de escucha, entonación y otros que sean necesarios para que Colombia se convierta en un país bilingüe. (PI)

El profesor nativo nos ayudó a mejorar la pronunciación y a aprender de otras culturas $(E)$

En cuanto al papel de los docentes en el proceso de aprendizaje, algunos estudiantes manifestaron que una de los resultados de la implementación del CSI y de los DBA sus docentes eran más creativos y daban lo mejor para brindar clases más dinámicas y divertidas, sin embargo, otros comentaron que a sus docentes les faltaba capacitación adecuada para dar sus clases adecuadamente. Los siguientes fragmentos ejemplifican la opinión de un estudiante:

Los docentes deberían estar más capacitados para ensenar inglés $y$ especialmente a estudiantes que no entienden y además usar herramientas más didácticas que hagan que el aprendizaje del inglés sea más fácil. $(E)$

Estas respuestas ciertamente brindan información que corrobora que los estudiantes desean tener docentes que los guíen y apoyen en el proceso de aprendizaje con prácticas pedagógicas ajustadas a sus necesidades, nivel lingüístico y contexto, como lo plantea también Gómez (2017) respecto a la necesidad de una preparación de los docentes acordes con la necesidad real del contexto colombiano.

\section{CSI Y DBA COMO APOYO AL DISEÑO, ADAPTACIÓN E IMPLEMENTACIÓN CURRICULAR}

Los resultados del estudio confirmaron que la mayoría de los docentes reconocieron que el CSI es una herramienta útil para promover un sentimiento de identidad global y nacional. El contar con una propuesta curricular a nivel nacional puede haber contribuido a que los docentes perciban el currículo y los derechos básicos como una manera de ir más allá de sus contextos locales y explorar las esferas nacional e internacional. Los docentes y directivos reconocieron que el currículo apoyó el uso de un enfoque curricular transversal lo cual significa que ellos han estado haciendo grandes esfuerzos para implementarlo teniendo en cuenta varias áreas del conocimiento. Además, el CSI contribuyó a fomentar el aprendizaje de sus estudiantes acerca y a través del idioma al mismo tiempo. Aunque algunos docentes expresaron que el CSI era muy ilustrativo, otros manifestaron que hubo dificultades al momento de adaptar el enfoque metodológico sugerido pues los docentes estaban acostumbrados a estrategias tradicionales basadas en lectura y escritura que poco consideraban factores contextuales y estrategias como los enfoques basados en tareas comunicativas y en proyectos, pues su labor se veía afectada por factores como el alto número de estudiantes en el aula, la falta de recursos y las limitantes de tiempo en el currículo, lo cual ratifica a Fandiño, Bermúdez and Lugo (2012) quienes sostienen que no se puede culpar a los docentes de los bajos niveles de desempeño de los estudiantes dadas las condiciones mencionadas. Los docentes también resaltaron que el CSI afectó en gran medida la forma en la que ellos planeaban sus 
clases, así como se puede notar en el siguiente fragmento que refleja el punto de vista de un docente:

Fue difícil trabajar con el currículo sugerido, pero luego noté que fue útil $y$ obtuve buenos resultados con los estudiantes. (D).

Los docentes y directivos también mencionaron la flexibilidad y adaptabilidad como características importantes del CSI las cuales les permitieron a las IE llevar a cabo una revisión de los componentes del Proyecto Educativo Institucional (PEI) para actualizar los modelos y enfoques pedagógicos en la clase de inglés e incorporar en ellas nuevas prácticas que oxigenaron el proceso de aprendizaje. Por otro lado, algunos docentes y directivos hablaron acerca de algunas dificultades durante el proceso de implementación del CSI y los DBA en sus instituciones, en lo referente al nivel de competencia esperado por el MEN el cual ellos consideraron demasiado exigente para las condiciones reales de las aulas de clase colombianas, especialmente por el tamaño de los grupos, la limitación en recursos y tiempo, la falta de capacitación continua de docentes y las múltiples funciones que ellos deben cumplir en su quehacer diario (Fandiño, Bermúdez \& Lugo, 2012). Un coordinador expreso lo siguiente:

Nosotros revisamos el CSI a fondo y encontramos que el nivel lingüístico propuesto en el SEC fue demasiado alto para nuestros estudiantes y por lo tanto decidimos seleccionar solo unas cuantas cosas para nuestras clases. (C)

\section{LIMITACIONES E INQUIETUDES EN LA IMPLEMENTACIÓN DEL CSI Y LOS DBA}

Otro de los resultados arrojados por el análisis de datos muestra que un alto número de docentes señaló que el MEN debería ofrecer más sesiones de capacitación y seguimiento a través de redes académicas para evaluar la implementación del currículo y recibir retroalimentación constate y valiosa de personas externas. Otros docentes fueron más allá y manifestaron que la actual política de bilingüismo fue una estrategia para transformar la vida de sus estudiantes y por supuesto su futuro pero que requiere más seguimiento y acompañamiento, lo mismo que inversión para darle continuidad a los procesos de desarrollo profesional de los docentes.

Por otro lado, otros docentes manifestaron que Ios DBA no fueron útiles como herramientas de evaluación. Una de las razones mencionadas fue el hecho de que las indicaciones brindadas por el MEN para que los docentes y estudiantes conocieran los DBA se basaron principalmente en diálogos y monólogos, los cuales no estaban alineados a los tipos de evaluación usadas por los docentes tales como exámenes escritos de unidad o de final de periodo, las pruebas externas estandarizadas y la integración de las cuatro habilidades. El siguiente fragmento muestra lo que un docente dijo acerca del SEC y los DBA juntos:

Los DBA fueron fáciles de implementar y se pudieron usar en todas las clases, pero los contenidos del CSI y el nivel requerido para enseñar algunos temas no estaban alineados con la realidad. Los estudiantes no comenzaban el grado 6 con un nivel $A$ y algunas veces ellos incluso terminan alcanzando apenas un nivel $A 1$. (D)

Otra limitante que fue mencionada en repetidas oportunidades tiene que ver con el número de horas semanales asignadas para la clase de inglés; la mayoría de los docentes y coordinadores mencionaron que los estudiantes por lo general recibían tres horas de clase a la semana, lo cual consideraban muy poco para alcanzar los objetivos y tener éxito en el aprendizaje del idioma. Con el fin de dar un ejemplo de este punto de vista un docente señalo lo siguiente: 
Ese número de horas no fue suficiente para lograr nuestros objetivos de clase. La mayor dificultad que tenemos en la implementación del currículo fue el número de horas por grupo asignadas para la materia y muchas veces algunas de estas se perdían debido a la programación de actividades extracurriculares planeadas por la institución educativa cada año o también por los días festivos y por consiguiente el tiempo para las clases de inglés no fue suficiente para el completo desarrollo del programa. (D)

Con respecto a los materiales y recursos tales como el English kit de los docentes y los libros de los estudiantes, el análisis de datos permite afirmar que los docentes efectivamente habían hecho uso de los materiales proporcionados por el MEN, sin embargo, algunos de ellos compartieron sus inquietudes acerca de las dificultades experimentadas por sus estudiantes para completar las actividades debido a su bajo nivel lingüístico. Un docente menciono su inquietud frente a esto:

Hubo temas específicos que fueron muy difíciles para los estudiantes teniendo en cuenta su nivel de competencia y por lo tanto fue necesario retomar algunos conceptos básicos de tal manera que dichos temas se ajustaran a las estructuras gramaticales y conocimiento de vocabulario de los estudiantes. (D)

De la misma manera, 15 de los 61 docentes mencionaron que mientras que los textos English Please! Fast Track no estaban completamente alineados a los BLR y al CSI, el contenido del texto Way to go! sí era consistente con estos documentos, lo que evidencia que los docentes exploraron los textos y sacaron conclusiones acerca de la relación entre los contenidos y los DBA y el CSI siendo recursivos y creativos en la adaptación de dichos materiales a las realidades de sus aulas de clase.
En cuanto a los recursos tecnológicos, el $39 \%$ de los docentes manifestaron que ellos usaban estas herramientas en clase; muchos docentes expresaron que todas las IE deberían tener conectividad para permitirles a los estudiantes completar las actividades en línea y explorar los sitios web recomendados en los textos. Específicamente, ellos le solicitaron al MEN diseñar $y$ ejecutar una plataforma virtual que apoyara las actividades incluidas en los textos. Otra recomendación fue la de adaptar aulas especiales en las IE para la enseñanza y aprendizaje del inglés con tecnología y, por consiguiente, se espera mayor inversión por parte del MEN.

\section{RECONOCIMIENTO DEL CSI COMO UN MEDIO DE CONTEXTUALIZACIÓN}

Con respecto a las temáticas abordadas en el currículo, la mayoría de los estudiantes y docentes compartieron comentarios favorables relacionados con la utilidad de dichos temas; ellos manifestaron que estos temas fueron útiles para aumentar la conciencia de los estudiantes acerca del medio ambiente, la paz en sus comunidades, cuidado personal y la sensibilización intercultural. Al mismo tiempo, los docentes expresaron que estos temas les ayudaron a aprender a través de discusiones relacionadas con el mundo de los adolescentes tales como sus rutinas de cuidado personal $y$ tendencias culturales y sociales tales como piercings, tatuajes y deportes extremos. De esta manera, las clases direccionadas a través del CSI también les permitieron a los estudiantes adolescentes revisar varios temas basados en sus necesidades e intereses por medio del uso del inglés como lengua extranjera. Desde la perspectiva de los docentes, éstos mencionaron que se esforzaron por brindarle a sus estudiantes lecciones enfocadas en los derechos humanos, inclusión y convivencia ciudadana y de esta manera estar alineados con las iniciativas 
nacionales en materia de ciudadanía, atención a la diversidad y promoción de procesos de paz.

\section{EXPECTATIVAS POR ESTRATEGIAS DE EVALUACIÓN MENOS TRADICIONALES}

Cuando se les preguntó acerca de la evaluación, la mayoría de los participantes estuvo de acuerdo en que los procedimientos de evaluación predominantes fueron los exámenes de periodo (85\%) y los exámenes finales $(80 \%)$. Estos resultados sugieren que las IE les brindan a los estudiantes principalmente evaluaciones que miden su progreso al final de una unidad o un curso por medio de una nota; el $60 \%$ de los estudiantes dijo que los portafolios fueron la técnica de evaluación menos usada. Algunos de los participantes manifestaron que los docentes apoyaban en gran medida las competencias del examen nacional estandarizado Saber-11 (ICFES, 2018) de tal manera que los estudiantes desarrollaron con mayor frecuencia ejercicios $y$ actividades relacionadas con vocabulario y comprensión de lectura. De todas maneras, tanto estudiantes como docentes y directivos, resaltaron nuevamente la flexibilidad del CSI al proporcionarle a los docentes una variedad de herramientas para la evaluación que se derivan de los enfoques aplicados y que privilegian el proceso de aprendizaje y no el resultado, como lo explican estos docentes y estudiantes:

Las formas sugeridas de evaluar en el currículo sugerido, son variadas y distintas a las que usualmente utilizamos, fue interesante conocerlas e implementarlas (D)

\section{NECESIDAD DE CONTINUIDAD EN LAS POLÍTICAS DE BILINGÜISMO}

Otra inquietud mencionada reiterativamente por los participantes en el estudio, es la necesidad de que el gobierno le dé continuidad a la implementación de las políticas de bilingüismo, pues consideran que con el cambio de gobiernos se interrumpen los procesos, las inversiones y las iniciativas que quedan abandonadas a la voluntad de los docentes que, por la falta de apoyo y acompañamiento, las dejan rezagadas $u$ olvidadas en el mar de tareas que deben cumplir; unos docentes manifestaron:

EI MEN debe continuar con las políticas de bilingüismo ya que estas le permiten a los estudiantes de IE públicas competir con las privadas y por lo tanto es una oportunidad para tener materiales de inglés con información acerca del país o departamento. (TS)

Es necesario que el Gobierno continúe promoviendo el bilingüismo por medio de capacitaciones a los profesores de inglés y continúe brindando herramientas para tener mejores resultados (D)

Esto sugiere que los docentes son conscientes de la importancia que las políticas de bilingüismo tuvieron en sus prácticas pedagógicas y su conexión con los contextos de los estudiantes, sin embargo, se sienten solos en su implementación y consideran que no se les da la fuerza suficiente para mantener en el tiempo e instalarse de manera más prolongada en los diferentes contextos educativos para lo cual se requiere voluntad política e inversión.

De otro lado, coordinadores, docentes, y estudiantes coinciden en que estas políticas son importantes y más fructíferas si se implementan desde los niveles de enseñanza preescolar y primaria, pues desde los primeros años se deben proporcionar a los niños la oportunidad de aprender la lengua extranjera, lo que facilita también el proceso en los niveles de secundaria y media; el siguiente fragmento ilustra esta opinión:

EI MEN debe implementar la enseñanza del inglés desde la escuela primaria ya que los docentes afirman que el 
aprendizaje del inglés desde temprana edad puede ayudar mucho a lograr los objetivos de ser competente en inglés $y$ que, aunque algunas instituciones educativas ofrecen clases de inglés a este nivel debería haber más horas. (C)

Los comentarios presentados sugieren que los participantes son conscientes de la importancia de comenzar el proceso de aprendizaje del inglés desde temprana edad, y aplauden cambios recientes en los que las instituciones han empezado a ofrecer el idioma extranjero en la escuela primaria y el MEN ha promovido la implementación de un currículo para la enseñanza del inglés desde preescolar hasta 5to grado (MEN, 2017).

\section{CONCLUSIONES}

Este estudio estableció las perspectivas de los estudiantes, docentes y directivos con respecto a las políticas de bilingüismo representadas en el CSI y los DBA y la forma como éstos se han incorporado en las prácticas pedagógicas en cinco regiones del país, con las cuales los investigadores llegamos a las siguientes conclusiones:

La implementación del CSI y los DBA en las IE públicas de Colombia ha afectado positivamente los procesos de enseñanza-aprendizaje del inglés en las aulas ya que los docentes han realizado ajustes y adaptaciones en el currículo para alinearse con las políticas y tendencias nacionales e internacionales. Esto representa beneficios muy significativos en la metodología que favorece la interacción y el desarrollo del idioma, junto con un mejor uso de recursos y enfoques de los cuales se destaca el Enfoque Basado en Tareas con el más usado y que les ofrece a los docentes una gran variedad de posibilidades para la planeación de sus lecciones, al igual que los Enfoques Basados en Proyectos y Problemas que aunque requieren mayores esfuerzos y más recursos y tiempo para ser implementados también brindan gran variedad metodológica al proceso.

El conocimiento de los contenidos y objetivos del CSI y los DBAes crucial en el logro de las políticas nacionales de bilingüismo; los estudiantes, docentes y directivos pueden ver en estos documentos un camino para implementar sus currículos de inglés para alcanzar los estándares nacionales y responder a las necesidades y expectativas de sus comunidades; la difusión de estas políticas en el quehacer diario de las aulas de clase de inglés eleva los niveles de interés entre los estudiantes pues al establecer metas claras de aprendizaje se mejoran también los niveles de atención y compromiso en las tareas realizadas.

Aunque las propuestas pedagógicas del CSI y los DBA, incluyendo el Programa de Expertos Nativos, apoyan el aprendizaje del idioma incluyendo las cuatro habilidades con una contextualización significativa respondiendo así a las necesidades e intereses de los estudiantes, la lectura sigue siendo la habilidad más importante debido a la administración de examen Saber-11; este factor constituye un reto para directivos y docentes pues debe buscarse un balance entre la evaluación formal del sistema educativo y aquella evaluación formativa y flexible que el SEC propone, por eso es importante que los directivos a nivel ministerial tengan en cuenta las voces de las comunidades para los ajustes necesarios en la evaluación estándar del sistema educativo para hacerla coherente con las prácticas propuestas en el CSI y los DBA.

La orientación transversal del CSI y los DBA es una gran contribución para la planeación de lecciones e implementación en las IE públicas colombianas debido a que esto ayuda a que los docentes y estudiantes relacionen la clase con los temas que se tratan en cada sesión; de igual manera, los temas de discusión relacionados con la edad de los estudiantes los motiva hacia 
el proceso de aprendizaje del idioma así como hacia las iniciativas asociadas al Programa de Nacional de Inclusión, los temas ambientales y el Proceso de Paz.

Aunque la provisión de materiales tales como los textos, cuadernos de trabajo, herramientas en línea y recursos audiovisuales buscan mejorar el proceso de enseñanza-aprendizaje dentro de las políticas del CSI y los DBA, los IE públicas colombianas necesitan recibir suficiente infraestructura de TIC, conectividad y capacitación docente con el fin de tomar ventaja de la gran cantidad de posibilidades que ofrece el MEN.

A pesar de las muchas posibilidades propuestas por el SEC en términos de evaluación del idioma, aún permanecen algunas prácticas tradicionales, especialmente las relacionados con la evaluación sumativa (quizzes, exámenes parciales y finales, exámenes estandarizados, etc.); es importante continuar trabajando a nivel gubernamental para la adaptación de las estrategias de evaluación del sistema educativo a las prácticas de aula de manera que se alcancen resultados más ajustados a la realidad de las mismas.

En general, podemos concluir que a pesar de las muchas debilidades relacionadas con limitaciones de tiempo, contenido, evaluación o infraestructura TIC, las respuestas en las encuestas y entrevistas reportaron que los participantes se mostraron satisfechos con el apoyo del MEN a través los programas de bilingüismo en el país, especialmente, el gran esfuerzo hecho para recortar la brecha entre el aprendizaje del inglés en las IE públicas y privadas, al igual que con el propósito de brindarle a la comunidad educativa una propuesta curricular abierta y flexible.

\section{REFERENCES BIBLIOGRÁFICAS}

Bakić, A. \& Škifić, S. (2017). The relationship between bilingualism and identity in expressing Emotions and thoughts. Íkala, 22 (1), 33-54. https://doi. org/10.17533/udea.ikala.v22n01a03

Bonilla, C. \& Tejada, I. (2016). Unanswered Questions in Colombia's Foreign Language Education Policy. Profile: Issues in Teachers' Professional Development, [S.I.], v. 18, n. 1, p. 185-201, Jan. 2016. ISSN 22565760. Available at: https://revistas. unal.edu.co/index.php/profile/article/ view/51996/54364>. Date accessed: 29 feb. 2020. doi:https://doi.org/10.15446/ profile.v18n1.51996.

Burns, Ann. (2001). Collaborative Action Research for English Language Teachers. Cambridge: Cambridge University Press.

Creswell, J. (2009). Research Design: Qualitative, Quantitative, and Mixed Methods Approaches. Los Angeles : Sage.

Fandiño, Y.; Bermúdez, J. \& Lugo, V. (2012). Retos del Programa Nacional de Bilingüismo. Colombia Bilingüe. Educación y educadores. 15 (3), 363381.

Fandiño, Y \& Jiménez, J. (2012). Bilingüismo: definición, perspectivas y retos. Revista Ruta Maestra, (16), 43-50.

Freeman, D. (1998). Doing Teacher-Research: From Inquiry to Understanding. NY: Heinle and Heinle Publisher.

Gómez, M. (2017). Review and analysis of the Colombian foreign language Bilingualism policies and plans. HOW, 24(1), 139-156. http://dx.doi. org/10.19183/how.24.1.343. 
González, A. (2010). English and English teaching in Colombia. Tensions and possibilities in the Expanding circle. In A. Kirpatrick (Ed.), The Routledge handbook of world Englishes, (pp. 332351). London: Routledge.

Herazo, J.; Jerez, S. \& Lorduy, D. (2012). Opportunity and incentive for becoming bilingual in

Colombia: Implications for Programa Nacional de Bilinguismo. Íkala, Revista de Lenguaje y Cultura, 17(2), 199213. Retrieved March 1, 2020, from http://www.scielo.org.co/scielo. php?script $=$ sci arttext $\&$ pid $=$ S0123$\underline{34322012000200006 \& \text { lng }=e n \& \text { tlng=en. }}$.

Instituto Colombiano para el Fomento de la Educación Superior (ICFES) (2018). Informe nacional de resultados del examen Saber 11. Bogotá D.C. : ICFES.

Mackenzie, L. (2019). A critical evaluation of the social justice implications of the Colombian

government's English-Spanish bilingualism policies. Current Issues in Language Planning. DOI: $\underline{10.1080 / 14664208.2019 .1615743 .}$.

Ministerio de Educación Nacional. (1994). Ley General de Educación. Santafé de Bogotá : MEN.Ministerio de Educación Nacional. (1996). Resolución 2343. Santafé de Bogotá : MEN.

Ministerio de Educación Nacional. (2001). Ley 715. Santafé de Bogotá : MEN.

Ministerio de Educación Nacional, MEN. (2006). Estándares básicos de competencias en lenguas extranjeras: Inglés. Bogotá : MEN. Ministerio de Educación Nacional, MEN. (2014). Orientaciones para la implementación de proyectos de fortalecimiento del inglés en las entidades territoriales. Bogotá, CO:

Author. Retrieved from http://www. colombiaaprende.edu.co/html/ micrositios/1752 articles-315518 recurso 5.pdf

Ministerio de Educación Nacional. (2015). Decreto 230/2002. Chapter 1. Article 2. Bogotá,

Colombia: Author. Retrieved from https:// www.mineducacion.gov.co/1621/ articles-355154_foto_portada.pdf

Ministerio de Educación Nacional, MEN. (2016a). Pedagogical principles and guidelines:

Suggested English curriculum, 6th to 11th grades. Bogotá, CO: Author. Retrieved from http://aprende.colombiaaprende. edu.co/sites/default/files/naspublic/ Anexo\%2015\%20Pedagogical\%20 Principles\%20and\%20Guidelines.pdf

Ministerio de Educación Nacional, MEN. (2016b). Implementation Model of an English

Fellowship Programme. Bogotá, CO: Author. Retrieved from http://aprende. colombiaaprende.edu.co/sites/default/ files/naspublic/colombiabilingue/3\%20

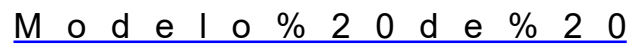
implementaci\%C3\%B3n\%20-\%20 Resumen\%20en\%20ingl\%C3\%A9s.pdf

Ministerio de Educación Nacional, MEN. (2016c). Suggested Curriculum Structure. Bogotá, CO: Author. Retrieved from

http://aprende.colombiaaprende.edu.co/sites/ default/files/naspublic/Anexo $\% 20$ 13\%20Suggested\%20Structure.pdf. 
Ministerio de Educación Nacional, MEN. (2017).

Mallas de Aprendizaje de Inglés para Transición a 5o. Bogotá,

CO: Author. Retrieved from

http://aprende.colombiaaprende.edu.co/sites/ default/files/naspublic/colombiabilingue/ dbacurriculo/cartillas mallas aprendizaje/Mallas $\% 20$ de $\% 20$ Aprendizaje.pdf

Ministerio de Educación Nacional. (2018). Colombia Bilingüe. Retrieved from

http://aprende.colombiaaprende.edu.co/es/ colombiabilingue/86689

Sánchez, A. \& Obando, G. (2008). Is Colombia ready for "bilingualism"? Profile Issues in Teachers Professional Development, (9), 181-196.

Trochim, W. \& Donnelly, J. (2008). The Research Methods Knowledge Base. United States : Cengage Learning.

Truscott de Mejía, A. M. (2006). Bilingual education in Colombia: Towards a recognition of languages, cultures and identities. Colombian Applied Linguistics Journal, (8), 152-168.

Truscott de Mejía, A. (2012). Reflections on English Language Teaching and Bilingualism in Colombia. Cuadernos del Caribe, 9(15), 23-30. Recuperado de https://revistas.unal.edu.co/index. php/ccaribe/article/view/40869/42567

Usma, J. (2009). Education and Language Policy in Colombia: exploring processes of inclusion, exclusion, and stratification in times of global reform. Profile Issues in Teachers' Professional Development, 11 (1), 129-143. 